\title{
Fixed Points of Some Classes of Nonexpansive Mappings
}

\author{
BranisLAV MiJAJLOVIĆ
}

\begin{abstract}
In this paper we proves the convergence of a convex sequence $x_{n}=\lambda x_{n-1}+(1-\lambda) f\left(x_{n-1}\right), \lambda \in(0,1)$, to a fixed point of the nonexpansive completely continuous operator in the normed $f_{\lambda}$-orbitally complete spaces with $\lambda$-uniformly convex sphere. Further we shall prove some fixed point theorems of the star-shaped sets.
\end{abstract}

\section{INTRODUCTION}

Let $X$ be a normed space. The mapping $f: X \rightarrow X$ where is called nonexpansive if it satisfies one of the following conditions:

1) $\|f(x)-f(y)\| \leq\|x-y\|$;

2) $\|f(x)-f(y)\| \leq \frac{1}{2}(\|x-f(x)\|+\|y-f(y)\|)$;

3) $\|f(x)-f(y)\|+\|y-f(y)\| \leq\|x-f(y)\|$.

Let $X$ be a vector space, $f: X \rightarrow X$ and $x \in X$. Let $\lambda \in(0,1)$ and $O_{\lambda}(x, f) \subseteq$ $X$ be a set defined by

$$
O_{\lambda}(x, f)=\left\{g_{0}(x, f(x)), g_{1}(x, f(x)), g_{2}(x, f(x)), \ldots\right\},
$$

where

$$
\begin{aligned}
& g_{0}(x, f(x))=x, g_{1}(x, f(x))=\lambda x+(1-\lambda) f(x), \\
& g_{n}(x, f(x))=g\left(g_{n-1}(x, f(x)), f\left(g_{n-1}(x, f(x))\right)\right) .
\end{aligned}
$$

Then $O_{\lambda}(x, f)$ is called convex orbit or $\lambda$-orbit of the point $x$ defined by $f$.

Let $(X, d)$ be a metric linear space, $f: X \rightarrow X$ and $\lambda \in(0,1)$. $X$ is $f_{\lambda}$-orbitally complete if each Cauchy's sequence from $O_{\lambda}(x, f)$ is convergent.

Large number of papers presents fixed point results for nonexpansive mappings (for $(L)$ type see: Browder [1], Karlowitz [4], Göhde [2], Kirk [5],...; for results on star-shaped sets see Reinermann's papers [8], [9]).

2000 Mathematics Subject Classification. 47H10, 54H25.

Key words and phrases. Normed $f_{\lambda}$-orbitally complete spaces with $\lambda$-uniformly convex sphere, Nonexpansive operator, $\lambda$-orbit, Fixed point, Extremal point, Star-shaped sets. 
2. The Convergence of the Convex Sequence

$$
x_{n}=\lambda x_{n-1}+(1-\lambda) f\left(x_{n-1}\right) \text { TО ТнЕ }
$$

Fixed Point of Nonexpansive $(L)$ Type Mapping

Let $\lambda \in(0,1)$. The normed space $X$ is the space with $\lambda$-uniformly convex sphere, if for each $\varepsilon>0$ there exists $\delta>0$, such that for all $x, y \in X$ from $\|x-y\|>\varepsilon$ follows:

$$
\|\lambda x+(1-\lambda) y\| \leq(1-\delta) \max \{\|x\|,\|y\|\} .
$$

For $f: E \rightarrow E$ we define $J(f, E)=\{x \mid f(x)=x\}$.

Lemma 2.1. Let $f: E \rightarrow E$ be a completely continuous linear operator, $E$ bounded subset of normed space $X$, and $J$ set of all solutions of the equation $x=f(x)$. Let

$$
R(J(f, E), \alpha)=\{x \mid x \in E, d(x, J(J(f, E)) \geq \alpha\} .
$$

Then for each $x \in R(J(J(f, E), \alpha)$ and each $\alpha>0$, there exists $\varepsilon=\varepsilon(\alpha)>0$ such that

$$
\|f(x)-x\|>\varepsilon
$$

and the $J(f, E)$ is a convex set.

The proof of the above Lemma can be found in [7].

Theorem 2.1. Let $\lambda \in(0,1)$ and $f: E \rightarrow E$ be a completely continuous operator, where $E$ is closed, bounded, and convex subset of the normed vector space $X$, which has $\lambda$-uniformly convex sphere. If $X$ is $f_{\lambda}$-orbitally complete space, and if $f$ satisfies the condition $(L)$, then the sequence $x_{n}=\lambda x_{n-1}+(1-\lambda) f\left(x_{n-1}\right)$, $n \in N$ is convergent for arbitrary $x_{0} \in E$, and its limit is the solution of the equation $x=f(x)$.

Proof. From definition of the sequence $x_{n}$ and condition $(L)$, follows:

$$
\begin{aligned}
d\left(x_{n+1}, J(f, E)\right) & =\inf _{y \in J(f, E)}\left\|x_{n+1}-y\right\|= \\
& =\inf _{y \in J(f, E)}\left\|\lambda x_{n}+(1-\lambda) f\left(x_{n}\right)-\lambda y-(1-\lambda) y\right\| \leq \\
& \leq \inf _{y \in J(f, E)}\left(\lambda\left\|x_{n}-y\right\|+(1-\lambda)\left\|x_{n}-y\right\|\right)= \\
& =d\left(x_{n}, J(f, E)\right),
\end{aligned}
$$

and so the sequence of numbers $d\left(x_{n}, J(f, E)\right)$ is non-increasing.

Let $x_{1}, \ldots, x_{k} \in R(J(f, E), \alpha)$. Since the space $X$ has $\lambda$-uniformly convex sphere, then for any $y \in J(f, E)$, we have:

$$
\begin{aligned}
\left\|x_{2}-y\right\| & =\left\|\lambda\left(x_{1}-y\right)+(1-\lambda)\left(f\left(x_{1}\right)-y\right)\right\| \leq \\
& \leq(1-\delta) \max \left\{\left\|x_{1}-y\right\|,\left\|f\left(x_{1}\right)-f(y)\right\|\right\} \leq \\
& \leq 2 M(1-\delta),
\end{aligned}
$$

where $M=\sup _{t \in E}\|t\|$. 
Similarly, we can prove that

$$
\left\|x_{k}-y\right\| \leq 2 M \cdot(1-\delta)^{k-1} .
$$

So,

$$
d\left(x_{k}, J(f, E)\right) \leq 2 M \cdot(1-\delta)^{k-1} .
$$

From the triangle inequality of and the (L) condition, follows:

$$
2\left\|x_{i}-y\right\| \geq\left\|f\left(x_{i}\right)-f(y)\right\|+\left\|y+x_{i}\right\| \geq\left\|f\left(x_{i}\right)-x_{i}\right\| \geq \varepsilon
$$

for $i=1,2, \ldots, k$, and $y \in E$.

From inequality (2.1) follows

$$
2 M \cdot(1-\delta)^{k-1} \geq \frac{\varepsilon}{2}
$$

and so

$$
k \leq 1+\frac{\ln 4 M-\ln \varepsilon}{-\ln (1-\delta)} .
$$

The sequence $\left\{d\left(x_{n}, J(f, E)\right)\right\}_{n \in N}$ is non-increasing for

$$
n>1+\frac{\ln (4 M)-\ln (\varepsilon)}{-\ln (1-\delta)} \quad \text { and } \quad d\left(x_{n}, J(f, E)\right)<\alpha .
$$

So

$$
\lim _{n \rightarrow \infty} d\left(x_{n}, J(f, E)\right)=0 .
$$

From (2.2) follows that for each $\beta>0$ there exists $n_{0} \in N$ and $y_{0} \in J(f, E)$, such that $d\left(x_{n}, J(f, E)\right)<\frac{\beta}{2}$ and $d\left(x_{n_{0}}, y_{0}\right)<\frac{\beta}{2}$, for $n_{1}, n_{2}>n_{0}$. It follows

$$
\left\|x_{n_{1}}-x_{n_{2}}\right\| \leq\left\|x_{n_{1}}-y_{0}\right\|+\left\|y_{0}-x_{n_{2}}\right\| \leq \frac{\beta}{2}+\frac{\beta}{2}=\beta .
$$

So $\left\{x_{n}\right\}_{n \in N}$ is a Cauchy sequence. Since the space $X$ is $f_{\lambda}$-orbitally complete, this sequence is convergent in $E$. Let $\lim _{n \rightarrow \infty} x_{n}=\xi$. From complete continuity of the operator $f$ and the definition of the sequence $x_{n}$, we obtain that $\xi=$ $\lambda \xi+(1-\lambda) f(\xi)$ and $\xi=f(\xi)$.

Theorem 2.2. Let $\lambda \in(0,1)$ and $p \in\{2,3, \ldots\}$. Let $f: E \rightarrow E$ be a completely continuous operator, where $E$ is closed, bounded, and convex subset of the normed vector space $X$ which has $\lambda$-uniformly convex sphere. If $X$ is $f_{\lambda}^{p}$-orbitally complete space and for every $x, y \in E$ :

$$
\left\|f^{p}(x)-f^{p}(y)\right\| \leq\|x-y\|
$$

then the sequence

$$
x_{n}=\lambda x_{n-1}+(1-\lambda) f^{p}\left(x_{n-1}\right), \quad n \in N
$$

is convergent for an arbitrary $x_{0} \in E$. Its limit is common solution of the equations $x=f^{p}(x)$ and $x=f(x)$. 
Proof. The operator $f^{p}: E \rightarrow E$ is completely continuous and maps the closed, convex and bounded set $E$ into $E$. The operator $f^{p}: E \rightarrow E$ is nonexpansive. So from Lemma 2.1 and Theorem 2.1, follows that the sequence defined by (2.3) is a Cauchy's sequence in $f_{\lambda}^{p}$-orbitally complete space and converges to a fixed point of operator $f^{p}$. The $J(f, E)$ and $J\left(f^{p}, E\right)$ are convex sets and $J(f, E) \subseteq J^{p}(f, E)$, which implies that the sequence (2.3) converges to the common solution of the equations $x=f(x)$ and $x=f^{p}(x)$.

\section{Fixed Points and Star-Shaped Sets}

Fixed point result of nonexpansive mapping of type (L), defined on star-shaped subsets of Hilbert's spaces was given in [8].

Let $X$ be a linear space. The $A \subseteq X$, is star-shaped if there exists $a \in A$, such that for each point $x \in A \lambda a+(1-\lambda) x \in A, \lambda \in(0,1)$. The point $a$ is called the star of the set $A$. The point $x \in A, X$ is is called extremal point of $\mathbf{A}$ if from $x=\lambda x_{1}+(1-\lambda) x_{2}, x_{1}, x_{2} \in A$ follows that $x_{1}=x_{2}=x$.

Let $X$ be a vector space, $f: X \rightarrow X$ and $x, a \in X$. Let $\lambda \in(0,1)$ and $O_{\lambda}(x, f) \subseteq X$ be a set defined by

$$
O_{\lambda}(a, x, f)=\left\{g_{0}(a, f(x)), g_{1}(a, f(x)), g_{2}(a, f(x)), l \ldots\right\},
$$

where

$$
\begin{aligned}
& g_{0}(a, f(x))=x, g_{1}(x, f(x))=\lambda a+(1-\lambda) f(x), \\
& g_{n}(a, f(x))=g\left(g_{n-1}(a, f(x)), f\left(g_{n-1}(a, f(x))\right)\right) .
\end{aligned}
$$

Then $O_{\lambda}(a, x, f)$ is called convex $\lambda, a$-orbit of the point $x$ defined by $f$.

Let $f: X \rightarrow X$, where $(X, d)$ is metric linear space and $\lambda \in(0,1) . \quad X$ is $f_{\lambda}^{a}$-orbitally complete if each Cauchy's sequence from $O_{\lambda}(a, x, f)$ is convergent.

Theorem 3.1. Let $\lambda \in(0,1)$ and $f: E \rightarrow E$ be completely continuous operator, where $E$ is closed, bounded, and star-shaped subset of the normed space $X$, which is $f_{\lambda}^{a}$-orbitally complete. Then $a$ is the star of the set $E$. If 0 is an extermal point of the set $E$, and if $f$ satisfies the condition $(L)$ or the condition $(B)$, then operator $f$ has a fixed point which is the limit of sequence

$$
x_{n}=\lambda a+(1-\lambda) f\left(x_{n-1}\right),
$$

for any $x_{0} \in E$.

Proof. The Theorem will be proved only for operator which satisfies the condition (B). The proof of the Theorem is similar operator $f$ satisfies the condition (L). From condition (B) we obtain

$$
\left\|f\left(x_{n-1}\right)-f\left(x_{n}\right)\right\|+\left\|x_{n}-f\left(x_{n}\right)\right\| \leq\left\|x_{n-1}-f\left(x_{n}\right)\right\| .
$$

From the definition of the sequence $x_{n}$, follows:

$$
\begin{aligned}
& \left\|\left(x_{n}-\lambda a\right)(1-\lambda)^{-1}-\left(x_{n+1}-\lambda a\right)(1-\lambda)^{-1}\right\|+ \\
& \quad+\left\|x_{n}-\left(x_{n+1}-\lambda a\right)(1-\lambda)^{-1}\right\| \leq\left\|x_{n-1}-\left(x_{n+1}-\lambda a\right)(1-\lambda)^{-1}\right\|,
\end{aligned}
$$


which implies

$$
\left\|x_{n}-x_{n+1}\right\| \leq(1-\lambda)\left\|x_{n-1}-x_{n}\right\| .
$$

It follows that the sequence defined by (2.3) is a Cauchy's sequence. Let $\lim _{n \rightarrow \infty} x_{n}=$ $\xi$. From (3.1) follows $\xi=\lambda a+(1-\lambda) f(\xi)$, which implies

$$
\lambda(\xi-a)+(1-\lambda)(\xi-f(\xi))=0 .
$$

From (3.2) follows that

$$
\xi=a=f(\xi)
$$

because 0 is an extremal point of set $E$. So the sequence (3.1) tends to the fixed point $x=a=\xi$.

In [7] was proved that mapping $f: E \rightarrow E$, where $E$ is a closed and convex subset of $f_{\lambda}$-orbitally complete space $X$, which satisfies the condition

$$
\|f(x)-f(y)\| \leq q(\|x-f(x)\|+\|y-f(y)\|),
$$

has a fixed point for $q \in\left[0, \frac{1-\lambda}{2-\lambda}\right), \quad \lambda \in(0,1)$.

Theorem 3.2. Let $\lambda \in(0,1)$ and $E$ be bounded and closed subset of normed $f_{\lambda^{-}}$ orbitally complete space $X$. If 0 is a star of the set $E$ and $f: E \rightarrow E$ is completely continuous operator satisfying the condition

$$
\|f(x)-f(y)\| \leq \frac{1-\lambda}{2-\lambda}(\|x-f(x)\|+\|y-f(y)\|), \lambda \in(0,1),
$$

then operator $f$ has at least one fixed point.

Proof. From the boundness of the set $E$ follows that there exists the ball $B(0, r)$ of the radius $r>0$ and center 0 , which contains it.

The mappings $q \cdot \frac{2-\lambda}{1-\lambda} f$ satisfy the condition (3.3), because from (3.4) follows

$$
\begin{aligned}
\left\|q \cdot \frac{2-\lambda}{1-\lambda} f(x)-q \cdot \frac{2-\lambda}{1-\lambda} f(y)\right\| & \leq q \cdot \frac{2-\lambda}{1-\lambda} \frac{1-\lambda}{2-\lambda}(\|x-f(x)\|+\|y-f(y)\|) \leq \\
& \leq q \cdot(\|x-f(x)\|+\|y-f(y)\|)
\end{aligned}
$$

for each $q \in\left[0, \frac{1-\lambda}{2-\lambda}\right)$. Then there exists a fixed point $z(\lambda, q)$ of the mapping $q \cdot \frac{2-\lambda}{1-\lambda} \cdot f$, that is $q \cdot \frac{2-\lambda}{1-\lambda} \cdot f(z(\lambda, q))=z(\lambda, q)$.

Now there is

$$
\begin{aligned}
\|f(z(\lambda, q))-z(\lambda, q)\| & =\left\|f(z(\lambda, q))-q \cdot \frac{2-\lambda}{1-\lambda} \cdot f(z(\lambda, q))\right\|= \\
& =\left(1-q \cdot \frac{2-\lambda}{1-\lambda}\right)\|f(z(\lambda, q))\| \leq\left(1-q \cdot \frac{2-\lambda}{1-\lambda}\right) r
\end{aligned}
$$


If $q \rightarrow \frac{1-\lambda}{2-\lambda}$, then $\left(1-q \cdot \frac{2-\lambda}{1-\lambda}\right) \cdot r \rightarrow 0$. Hence, for each $\varepsilon>0$ there exists $z(\lambda, q)$ such that

$$
\|f(z(\lambda, q))-z(\lambda, q)\|<\varepsilon \quad \text { if there is } \quad q>\left(1-\frac{\varepsilon}{r}\right) \cdot \frac{1-\lambda}{2-\lambda} .
$$

Let $\varepsilon \in\left\{1, \frac{1}{2}, \frac{1}{3}, \ldots\right\}$. According to (3.6), for every $\varepsilon>0$ from

$$
q_{1}>\left(1-\frac{\varepsilon}{r}\right) \cdot \frac{1-\lambda}{2-\lambda}, q_{2}>\left(1-\frac{\varepsilon}{2 r}\right) \cdot \frac{1-\lambda}{2-\lambda}, \ldots
$$

that there exists a sequence of fixed points $y_{1}, y_{2}, \ldots$, such that

$$
\begin{aligned}
\left\|f\left(y_{1}\right)-y_{1}\right\| & <1 \\
\left\|f\left(y_{2}\right)-y_{2}\right\| & <\frac{1}{2} \\
& \vdots \\
\left\|f\left(y_{n}\right)-y_{n}\right\| & <\frac{1}{n}
\end{aligned}
$$

It follows

$$
\left\|f\left(y_{n}\right)-y_{n}\right\| \rightarrow 0
$$

when $n \rightarrow \infty$.

Since the operator $f$ is completely continuous, the sequence $\left\{f\left(y_{n}\right)\right\}_{n \in N}$ has at least one convergent subsequence $\left\{f\left(y_{n_{p}}\right)\right\}_{n_{p} \in N}$. Let $\lim _{n \rightarrow \infty} f\left(y_{n_{p}}\right)=\xi$. According to (3.8), we also have $\lim _{n \rightarrow \infty} y_{n_{p}}=\xi$, and it follows that $\|f(\xi)-\xi\|=0$. So, $\xi=f(\xi)$.

If the condition (3.4) of Theorem (3.2) is replaced by the condition $(K)$ following from the result of paper [3] and from the condition of Theorem (3.2), it can similarly be shown that, if $E$ is a subset of Banach's space $X$, the mapping $f: E \rightarrow E$ has at least one fixed point.

\section{REFERENCES}

[1] F. E. Browder, Nonexpansive nonlinear operators in a Banach space, Proc. Nat. Acad. Sci., 54(1965), 1041-1044.

[2] D. Göhde, Zum, Princip der kontraktiven Abbildung, Math. Hachr., 30(1965), 251-258.

[3] R. Kannan, Some results on fixed points I, Bull. Calcutta Math, Soc., 60(1968), 71-76.

[4] L. A. Karlovitz, Some fixed point results for nonexpansive mappings, Fixed Point Theory and Its Aplications, Academic Press, (1976), 91-103. 
[5] W. A. Kirk, A fixed point theorem for mappings which do not increase distance, Amer. Math. Montly, 72(1965), 1004-1006.

[6] M. A. Krasnoselskij, Two remarks on the method of successive approximations, Uspehi. Mat. Nauk, 10(1955), 123-127, (Russian).

[7] B. S. Mijajlović, Fiksne tačke, mere nekompaktnosti i prošireno konveksne funkcije, Doktorska teza, Filozofski fakultet, Niš, (1999).

[8] J. Reinermann, Fixed point theorems for nonexpansive mappings on starshaped domains, Ber. Ges. Math. Daten, Bonn, (1974).

[9] J. Reinermann and R. Schöneberg, Some results in the fixed point theory of nonexpansive mappings and generalized contractions, Fixed Point Theory and Its Aplications, Academic Press, (1976), 175-186.

[10] M. R. Tasković, Nonlinear Functional Analysis, part I: Fundamental elements of theory, Zavod za udžbenike i nastavna sredstva, Beograd (1993), 792 p.p. Serbian-English summary: Comments only new main results of this book. Vol. 1 (1993).

FACUlTy of Teacher Education Milana Mijalkovića 14 35000 JAGODINA

SERBia And Montenegro 